\title{
Arbor
}

\section{Documento I: Discurso leído por Jose Echegaray ante la Real Academia de Ciencias Exactas, Física y Naturales en su recepción pública el once de marzo de 1866}

La honra que de esta muy respetable Academia he recibido, honra tan superior a mis méritos, si méritos hay en mí, que no como justa recompensa sino como bondadoso estímulo debo considerarla, me impone grandes deberes. No abrigo la esperanza de cumplirlos cual corresponde al nombre ilustre de esta corporación, al saber de sus individuos y al brillo de la ciencia moderna en nuestra patria, porque fueran en mí tales esperanzas locas ambiciones; más si con buen deseo, con celo infatigable pueden suplirse en algo dotes más elevadas, con uno y otro, que es cuanto de mi voluntad depende, procuraré mostrar mi profundo agradecimiento por este elevado título que sin merecer recibo, y que jamás esperé.

De estos sagrados deberes hoy cumplo uno, y solo por cumplirlo voy a ocupar la atención de la Academia: no la merece mi persona; pero la importancia del punto que he escogido, los arduos problemas que encierra y su inmensa trascendencia para el porvenir, le hacen digno de estudio y meditación: que al fin es la ciencia, por más abstracta que en sus concepciones a primera vista parezca, germen fecundo de progreso para pueblos, enérgico purificador del alma, luz que alumbra a la humana inteligencia con divinos resplandores.

Voy a ocuparme de la historia de las Matemáticas puras en nuestra España; y entiendo por Matemáticas puras la ciencia eminentemente racional, no la Física, ni la Astronomía, ni todas aquellas que, si bien acuden al análisis algebraico o geométrico como a poderoso auxiliar, son por su naturaleza, y por el carácter de los fenómenos que estudian, verdaderas ciencias de observación. Mas aquí he menester de toda mi fuer- 
za de voluntad para no pagar tributo a sentimientos que, bien lo sé, son nobles y naturales en su origen, pero que deben, como todo lo humano, tener un límite, y es este límite la verdad severa de los hechos; para ahogar en mí el grito del amor patrio, que a todo trance quisiera hoy hacerme decir alabanzas que la Historia con su elocuente voz me afirma que fueran inmerecidas; para no dejarme, en fin, arrastrar por la costumbre que en actos como este exige siempre glorias nacionales que referir con épica entonación, no frías y severas lecciones que leer tristemente en el incorruptible libro de la historia. Espero, sin embargo, vencer estos impulsos y sin mas norte que la verdad, no perdonando medio para hallarla, ni oscureciéndola nunca por mucho qué mortifique mi orgullo nacional, voy, repito, a trazar a grandes rasgos la historia de las Matemáticas en nuestro país.

La ciencia como la luz, y luz es en verdad del espíritu, aparece en los primeros tiempos históricos allá en Oriente, y girando magestuosa como astro disipador de tinieblas, avanza hacia el Occidente sumido por entonces en densas sombras. Difícil es decir con certidumbre lo que fueron en Egipto las Matemáticas puras, y tampoco para nuestro objeto importa mucho tal investigación, por más que bajo otro punto de vista yo reconozca su importancia; pero hay razones, y razones poderosas, para creer que si ya existían en aquellos tiempos la Aritmética y la Geometría, más que por derecho propio eran cultivadas como auxiliares de la Astronomía, de la Topografía, de la Mecánica y de la Arquitectura. En el Egipto sin embargo recoje la Grecia, según Aristóteles y Platón, los primeros rudimentos de la Aritmética y de la Geometría, si bien Estrabon, Porfirio y otros historiadores dividen entre egipcios y fenicios el alto honor de haber dado enseñanza a los primeros filósofos griegos. Sea una u otra la opinión más fundada, ello es que la ciencia pura que en aquellos antiguos pueblos orientales apenas era germen confuso y débil, fecundado por el creador genio helénico, crece y se extiende, se eleva a magestuosa altura con Thales, Pitágoras, Platón y mil otros filósofos, que en gracia a la brevedad no citaré.

Tiende Alejandro su vencedora espada sobre el, para nosotros, remoto Oriente; acosa y atropella y vence pueblos y pueblos; amasa con la sangre de los que destruye y la espantada vida de los que quedan un colosal imperio; y cuando el conquistador muere, y la conquista se deshace, y el imperio se derrumba, aparece en Egipto el nuevo e ilustre reino de los Ptolomeos, en el que renace el espíritu matemático de la antigua Grecia; y tanto crece y se agiganta, y a tan sublime altura llega, que siglos y siglos pasarán, y todos los prodigios de la moderna Geometría, y todo el ge- 
nio de Girard, de Simson, de Chasles, serán necesarios para comprender los maravillosos descubrimientos del inmortal Euclides en sus enigmáticos Porismos.

Y aquí la ciencia alcanza nueva vida: la escuela Alejandrina, Euclides, Eratósthenes, Apolonio con sus peregrinas y sutilísimas invenciones sobre las cónicas, Diofanto, creador del Algebra según muchos, y allá en Sicilia el colosal genio de Arquímedes, que hace de Siracusa la rival de Atenas y de Alejandría, y más tarde mil ilustres geómetras que estienden y perfeccionan y comentan las portentosas obras de sus predecesores y maestros, noble raza que, hacia el siglo IV de nuestra era, termina en Theon, y lanza el último y doliente suspiro con la desventurada y poética Hypathía, bárbaramente asesinada en las calles de Alejandría por sanguinarios y miserables fanáticos, preludio siniestro del odio mortal que más tarde y siempre había de profesar el fanatismo religioso a la ciencia, tantos y tantos inmortales ingenios forman y cierran, porque en ellos concluye el ciclo verdaderamente heroico de las Matemáticas, y dejan radiante de gloria e inundada de luz, que catorce siglos apenas harán palidecer, la historia de aquella maravillosa edad.

La Grecia, patria de héroes, de artistas, de filósofos, fue también tierra privilegiada de grandes geómetras, y de esta suerte compendió al hombre en las más nobles manifestaciones de su esencia inmortal. No así la Roma antigua: fue Roma en aquellos tiempos lo que ha sido España en el renacimiento y en la edad moderna. Buscad en Roma como en España todas las glorias, y las hallareis todas, como dice Libri, menos una: la de haber dado al mundo un geómetra de primer orden; un Euclides como Grecia, un Arquímedes como Sicilia; un Harriot, un Wallis, un Newton como Inglaterra, un Viete, un Descartes, un Fermat, un Laplace como Francia; un Lagrange como Italia; un Euler, un Leibnitz como Alemania; un Abel como Noruega. Quizá hallareis nombres respetables; respetables, sí, pero grandes geómetras, genios potentes, de esos que graban como con sello divino su nombre inmortal en un siglo y le hacen suyo, no, no los busqueis, que ni en Roma ni en España podreis hallarlos; y doy a mi patria en su aflicción ilustre compañera , Roma, que es cuanto hacer por ella puedo.

Sólo en el siglo V aparece un geómetra que merezca citarse: Manlius Severinus Boetius, matemático distinguido en verdad, gran conocedor de la ciencia griega, inteligente comentarista; su nombre será pronunciado siempre con respeto; pero no alcanzó la talla heróica de los Arquímedes y de los Euclides, ni pudo abrir con su genio, como Viete, como Descartes, como Newton, como Leibnitz, nuevos horizontes al ambicioso pensa- 
miento.

Pasan los tiempos, y mientras innumerables naciones bárbaras caen como granizo impulsado por viento de tempestad sobre el mundo romano, una nueva raza, noble, grande, injustamente tratada no pocas veces por escritores cristianos, aparece en la historia. Luchan los árabes, vencen y conquistan, y fundan un gran imperio, que como creciente marea se estiende desde la India hasta el pie de las elevadas montañas de Asturias, estrellando en ellas con impotente furia el tremendo oleaje de sus vencedoras armas. Raza noble y grande, repito, que si en el delirio de la lucha (siglo VII) entrega a las llamas la gran biblioteca alejandrina -bárbaro rasgo sin embargo, que respetables historiadores ponen en duda, porque no fueron los árabes en su sentir, sino fanáticos de muy distinta religión, los que con funesta ceguedad destruyeron aquel gran, monumento del antiguo saber, -acojen en cambio con afan todos los restos de la ciencia helénica, y conservan con amoroso cuidado tan rico depósito. Los árabes introducen en Europa las cifras índicas; cultivan la Aritmética y la Geometría; traducen y esparcen en el mundo cristiano los libros griegos; perfeccionan, y según algunos historiadores crean el Álgebra o Almucabala y la Trigonometría; y fundan por do quiera escuelas, academias y bibliotecas, que son brillantes faros de purísima luz en aquellos siglos medios de profundas y densas tinieblas.

Si este continuo flujo de razas y de pueblos, que uno tras otro caen sobre los países occidentales, es ley providencial que de esta suerte, y bajo la ruda forma de la conquista, impone a una nación, ya los dioses, ya la ciencia o el arte, ya la forma política de otra más adelantada, o es más bien terrible azote que priva al pueblo vencido de su libertad, y ahoga en él gérmenes fecundos de vida que en siglos venideros hubieran dado origen a una nueva y espontánea, no copiada y servil, civilización, es problema de todo punto ageno a mi propósito; ello es lo cierto que a los árabes, que al África, a España y a Sicilia trajeron su saber, y el saber aún mayor de la Grecia, debe el mundo cristiano los fundamentos de las ciencias matemáticas que son hoy nuestro orgullo y nuestra gloria.

Ya a fines del siglo VIII, esterminados los Omeyas, y triunfantes los Abasidas, termina el período de la conquista, y comienzan a saborearse las dulzuras de la paz; el gusto por las ciencias se desarrolla entre los árabes; sus feroces instintos se suavizan; el arte y la poesía les elevan a nueva vida; y mil ilustres ingenios hacen de Bagdad, la Atenas del Oriente. Mohamed Ben Musa, o hijo de Moisés, que Leonardo de Pisa y el célebre Tartaglia suponen ser el inventor del Algebra, hipótesis falsa, pero que prueba la elevada opinión que del geómetra árabe tenían: Thébit Ben 
Corrah, fecundo y eminente matemático; Hassen, uno de los tres hijos de Musa, de quien se dice que resolvió problemas geométricos que hubieran sido el asombro de la antigüedad; Alkindi, a quien coloca Cardano, siquiera sea hiperbólico el elogio, entre los doce más claros ingenios que hasta entonces hubiesen ilustrado al mundo, y tantos otros como la historia de aquellos tiempos cita, y cuyas obras, si de los autos de fe se salvaron, en el Escorial o en Toledo o en alguna otra biblioteca descansarán hoy, dan nombre y nombre glorioso a la raza oriental de que proceden.

España fue entonces; pero no la España cristiana, el centro del saber en Europa: en las célebres escuelas de Córdoba, de Sevilla, de Murcia y de Toledo, se enseñaba toda la ciencia acumulada durante tantos y tantos siglos en Oriente.

De todas partes, de Inglaterra, de Francia, de Italia, de Alemania, acudían estranjeros ganosos de saber, buscando entre los árabes espanoles los ricos tesoros de la codiciada ciencia. Alberto el Grande, Gerberto, Pedro el Venerable, Platón de Tívoli, Gerardo de Cremona, vinieron a esta nuestra tierra, a aprender lo que más tarde en las suyas enseñaron. Entonces tuvimos en ciencias matemáticas sabios ilustres; el Obispo Aiton, Josef, el renombrado Juan de Sevilla y otros varios, sin contar astrónomos, ni físicos, ni mecánicos, pues solo de la ciencia pura me ocupo; entonces Alfonso el X, rey de imperecedera memoria, se rodeaba de árabes y hebreos, y dejaba en sus tablas alfonsíes glorioso monumento al porvenir. Pero cuenta que aquellas nuestras glorias, son glorias de los árabes españoles; y si del pueblo enemigo renegamos; si , como redujimos a ceniza sus bibliotecas, reducimos a ceniza en el fuego de nuestro odio tradicional el recuerdo de tanto y tanto geómetra árabe; si, como arrojamos de nuestro suelo, que era también el suyo, a sus infelices descendientes, arrojamos de nuestra historia aquellas sus pasadas glorias, ninguna, que solo a nosotros pertenezca, nos queda.

Bien quisiera, señores, detenerme aún más en este glorioso período de nuestra historia científica; y como peregrino que desde deleitoso oásis ve estenderse ante su vista la inmensa y árida superficie del desierto, duda y vacila en abandonar el encantador jardín por la abrasada y silenciosa llanura, así dudo y vacilo en continuar esta rápida e imperfecta relación, porque silencio y soledad sé que he de encontrar en mi camino. Pero el tiempo apremia, vuestra benévola atención me obliga, y aun la materia misma me estimula a llegar rápidamente al término de esta mi tarea.

La edad media termina al fin; sucumbe Constantinopla; huyen los griegos del bajo imperio de la sangrienta cuchilla de los turcos; miles y miles de manuscritos, que mas tarde la imprenta reproduce y difunde por 
Europa, descubren nuevos e ignorados tesoros de la sabia antigüedad; y llega para las artes y las ciencias, lo que llaman los historiadores el renacimiento.

No he de detenerme a juzgar este gran acontecimiento histórico, cuya importancia sin embargo creo que algo se ha exajerado; porque diríase, si a ciertos escritores se creyese, que todo era sombras en Europa hasta que el imperio Bizantino se derrumbó, y que por la brecha que en las viejas murallas de Constantinopla abrieron los turcos, se escapó a torrentes la ciencia y el arte, hasta entonces por misteriosos conjuros en la mágica ciudad encerrados. No en verdad; la herencia de la antigua Grecia se dividió, y si una buena parte quedó en Bizancio, otra quizá mejor tocó a los árabes que desde el Eufrates al Tajo en su victoriosa marcha triunfalmente la trajeron, y a las naciones cristianas de Europa noblemente la entregaron. No fue el renacimiento repentino despertar de los pueblos occidentales; siglos y siglos de lucha y de trabajo; ilustres pensadores, profundos filósofos, inmortales aunque desconocidos artistas lo habían preparado lentamente; y en esta gran obra los árabes ocupan quizá el primer término, y se hacen dignos de la gratitud de los pueblos modernos y del imparcial aplauso de la historia. Si después se dividen, y en luchas intestinas se desgarran, y con razas ignorantes y feroces se mezclan, degenerando de esta suerte de su antigua grandeza, cruel castigo reciben por sus faltas; la Europa les rechaza a punta de lanza y a filo de espada hasta las fronteras de la barbarie, y cubre de soberbio desprecio a los descendientes de sùs ilustres maestros: ni acuso de ingratitud, ni absuelvo de culpa, más me conduelo de la suerte fatal que a este gran pueblo estaba reserváda.

El siglo.XIV fue casi estéril para los descubrimientos matemáticos; como si el mundo antiguo hubiera agotado toda su fuerza creadora en los Euclides, en los Arquímedes y en los grandes geómetras árabes, y cayese al fin rendido y estenuado; o como si la naturaleza, recojida en sí misma, condensase todas sus energías en misterioso y sublime trabajo, preparándose para aquel aborto gigante que dio al mundo un Newton, un Descartes, un Leibnitz, un Euler, un Lagrange y todos los admirables analistas de los siglos XVI, XVII y XVIII, reinan en Europa por largos años profunda calma, prolongado y solemne silencio.

En el siglo XV presenta ya Italia algunos geómetras eminentes: Leonardo Fibonacci, de Pisa, que aprendió el Álgebra o Ars-magna en sus viajes al África y a Oriente, había traido (siglo XIII) la nueva ciencia a su patria, y Lucas Paccioli, de Burgo, aunque bajo la forma imperfecta de tales tiempos, la estudia y la perfecciona y luchando con las dificultades de aquel bárbaro Algoritmo, supliendo siempre por el raciocinio directo las 
Documento I: Discurso leído ante la Real Academia...

elegantes y fecundas trasformaciones de nuestro análisis, resuelve problemas dificilísimos para entonces. Francisco Ghaligai; Pedro de Burgo; el español Ortega, residente en Roma; el célebre Leonardo de Vinci, genio sublime que a todas partes llega y en todo se muestra colosal; y por fin Regiomontano, que aplica en una obra bajo todos aspectos notable, el método algebráico a la resolución de triángulos; todos estos insignes matemáticos inician ya en el siglo XV el gran movimiento de los siglos venideros.

En el siglo XVI, la que podemos llamar evolución arábiga termina por completo, y la ciencia abandona a los pueblos orientales para venir a nuestra Europa, como sol que, dejando tras de sí tristes sombras avanza derramando luz y vida. En Italia aparece, más presto se estiende a Francia, Holanda, Alemania e Inglaterra; y si al principio solo se traducen, amplían y comentan las obras griegas, bien pronto, el nuevo espíritu geométrico se muestra, ensayando audaz sus antes ignorados y maravillosos recursos.

En Italia descubre Scipion Ferro, de Bologna, la resolución de las ecuaciones de tercer grado, pero muere sin publicar su descubrimiento, y el siempre desgraciado Tartaglia, de tosca educación pero inteligøncia noble y elevada, las resuelve a su vez; Cardan las perfecciona, y señala el caso irreducible; Ferrari resuelve las de cuarto; Bombelli penetra aún más en el estudio de unas y otras, y con gran sagacidad sospecha y casi adivina la engañosa apariencia de las tres raíces imaginarias, crea en Algebra notaciones abreviadas, y desarrolla por completo el cálculo de radicales.

En Alemania Werner, Rudolfs, Rethius, Stifels, precursor de Neper, y otros muchos, cultivan con toda la proverbial tenacidad germánica la gran ciencia.

Propagan en Francia la Aritmética y la Geometría Pelletier, Buteon y otros muchos escritores, estranjeros la mayor parte, pero que en la Universidad de París, metrópoli ya de las ciencias y las letras, se reunen.

Brillan en Bélgica, en Holanda y en los Países-Bajos Metius y Stevin; y hasta nuestra Península, antes de caer en el mortal abatimiento de los siglos venideros, hace un último esfuerzo, y da el postrero y angustioso adios a sus pasadas glorias con el castellano Juan Rojas, y con el ilustre portugués Núñez, eminente geómetra, que escribe una escelente obra de Algebra; que resuelve el, para aquella edad, dificilísimo problema del menor crepúsculo, problema que aun al genio poderoso de Bernoulli resiste por algún tiempo; que inicia la teoría de las loxodromas, y que se eleva como geómetra y como astrónomo a gran altura. 
El siglo XVI termina, más antes aparece el francés Viete, creador del Álgebra moderna, y precursor de los grandes genios del siglo XVII.

Nunca un hombre, sea cual fuere su talento, crea por sí solo y de una vez toda una ciencia: el Álgebra, como el cálculo infinitesimal, como todo descubrimiento importante, tiene su historia, sus precedentes, su crepúsculo por decirlo así. Al genio que organiza, condensa y da unidad, preceden otros hombres que acumulan materiales y preparan la gran obra, y así a Viete precede un gran período de lenta elaboración en Oriente; Diofanto, que resuelve difíciles problemas de análisis indeterminado; y los algebristas árabes; y Leonardo de Pisa; y Lucas de Burgo; y los italianos de los siglos XV y XVI; y muchos otros geómetras de este último siglo. Materiales había y abundantes, pero faltaba el arquitecto, y a Viete corresponde de justicia esta gloria. Él crea el Álgebra moderna, y por invención tan admirable abre a la ciencia nuevos y dilatados horizontes, que más se estienden cuanto más hacia ellos se avanza. ¿Y cuál la clave de esta portentosa invencion? Sencilla en estremo, trivial casi, sin relación lógica al parecer con sus maravillosos resultados: representar por letras los datos y las incógnitas de los problemas; he aquí todo. Y sin embargo, esta idea tan sencilla, ora es firmísimo punto de apoyo en que el pensamiento descansa al aplicar las leyes eternas de la lógica, ora rápida y poderosa locomotora, en que vuela audaz tras los principios generales de la cantidad y del orden.

Más en verdad, que si tales resultados no podían preveerse, pueden hoy esplicarse fácilmente.

La razón humana, en su desarrollo histórico, procede siempre elevándose de lo particular a lo general, de las relaciones sencillas a los grandes principios; y cuando de esta suerte se halla en posesión de una ley, es dueña absoluta de toda la esfera racional que esa ley domina, y de todo lo que en ella como en su unidad superior se halla encerrado. La sustitución de las letras, símbolos generales, a los números, es la sustitución de la gran categoría de la cantidad a tales o cuales cantidades particulares; y toda verdad que de este modo se demuestre, abarcará cuanto aquella categoría abarca. Por otra parte, si el raciocinio directo solo puede desarrollarse en serie lineal de silogismos, el Álgebra, al contrario, condensa en una espresión analítica todo un razonamiento, y al combinar muchas ecuaciones entre sí, o al someterlas a determinadas trasformaciones, no opera sobre sencillos silogismos, sino más bien sobre series estensísimas, que une y enlaza, por decirlo así, en múltiples dimensiones.

$\mathrm{El}$ álgebra es, respecto a la lógica ordinaria, lo que la colosal máquina de vapor a la primitiva palanca impulsada por el brazo del hombre. 
Documento I: Discurso leído ante la Real Academia...

Bien pronto, en efecto, se muestra el incalculable poder de la nueva invención. El mismo Viete la ensaya con gran talento, y obtiene importantes resultados: da unidad a la teoría de ecuaciones; las transforma y prepara; quita denominadores y radicales; descubre reglas para sumar o restar un número dado a las raíces de toda ecuación, y para multiplicarlas o dividirlas por un factor cualquiera; resuelve por este nuevo método las ecuaciones de segundo grado; estudia y descubre la composición de los coeficientes; construye las raíces de las ecuaciones de tercer grado; hace ver que este último problema depende del de la duplicación del cubo, o del de la trisección del ángulo; inicia la teoría de las secciones angulares; restaura la obra de Apolonio titulada de los contactos; aplica el análisis a la geometría; espresa el área de las curvas por series; a todas partes llega con su genio poderoso y su infatigable espíritu, y así termina dignamente el siglo de Tartaglia, de Cardan y de Ferrari.

Más por grande que haya sido el siglo XVI, le aventaja en mucho el admirable siglo XVII, siglo de jigantes, ante los que se inclina vencida la sabia antigüedad. En todas partes brotan a porfia y por decirlo así se atropellan eminentes geómetras, que eclipsan las glorias de los griegos y de los árabes.

En Italia Ricci, Valerio, Caravagio, Marchetti, Borelli, Mengoli, Torricelli, Vivían, discípulo del inmortal Galileo, y el eminente geómetra milanés Cavallieri, precursor de Newton y Leibnitz con su teoría de los indivisibles.

En Flandes Hudde, cuyos trabajos merecieron los elogios de Leibnitz; Van-Heuraet, que es el primer geómetra del continente que rectifica exactamente una curva; y Alberto Girard, que estudia los ángulos sólidos y la medida de las figuras esféricas, que inicia ocho años antes que Descartes la teoría de las raices negativas, y hace hablar a la esfinge de los porismos.

En Holanda Huygens, que crea la teoría de las evolutas, y por su genio profundo y sus admirables trabajos tan preciadas alabanzas mereció de Newton; y otros muchos geómetras que, con los Flamencos, son los primeros de Europa que comprenden y aplican la geometría Cartesiana.

En Bélgica Sluze y Gregorio de San Vicente, a quien considera Leibnitz como rival de Fermat y de Descartes.

En Suiza los Bernoulli, que huyendo como otros muchos de las crueles persecuciones del Duque de Alba, buscaron paz e independencia donde solo pueden hallarse, que es en los paises libres; y es triste ver como con nuestro despotismo político y con nuestra intolerancia religiosa, no 
contentos con ahogar al genio en nuestra patria, íbamos por Europa aventándolo ante nuestros sangrientos pendones.

En Dinamarca Roemer, que estudia las epicicloides.

En Francia Guldin, que da nombre a su ingeniosísima y profunda teoría sobre volúmenes y áreas.

Roberbal, que estudia con envidiable talento las parábolas de orden superior, la cicloide y otras muchas curvas, y resuelve el problema de las tangentes por la combinación de movimientos, idea que coincide bajo el punto de vista geométrico con la teoría de las fluxiones.

Desargues, eminente geómetra, que asienta los fundamentos de la moderna geometría trascendente, y descubre para las cónicas el fecundo teorema sobre la involución de seis puntos.

Bachet de Meziriac, que comenta la obra de Diofanto, y resuelve por vez primera, segun Lagrange, las ecuaciones indeterminadas de primer grado.

El digno rival de Descartes, el gran matemático Fermat, genio potente, infatigable, profundo, que estudia las espirales, las parábolas, la cuadratura de figuras curvilíneas, las cónicas la cicloide; que inicia la teoría de la eliminación, crea la de los números, resuelve el problema de los máximos y mínimos, y el de las tangentes, preparando de este modo el descubrimiento del cálculo diferencial; Fermat, repito, de cuya mente brotan a torrentes ideas, que por donde quiera esparce con la prodigalidad, del verdadero genio; y cuyos trabajos concisos siempre y a veces enigmáticos, son estudiados y comentados en el siglo XVIII por Euler y Lagrange, en innumerables Memorias que llenan las Academias de Petersburgo y Berlín.

Pascal, inteligencia superior, que a los 16 años escribe un admirable libro sobré las cónicas, y es mas tarde uno de los primeros geómetras de su época.

A fines del siglo, Rolle, que perfecciona la teoría de ecuaciones.

La Hire, célebre por sus trabajos geométricos.

El Marqués de l'Hopital, uno de los pocos que con Varignon, geómetra francés, y los Bernoulli, comprende en el siglo XVII el cálculo diferencial, y lo cultiva.

Y por último, gigante entre gigantes, el gran filósofo francés, el in mortal Descartes, cuyos bellísimos trabajos sobre curvas en general, áreas, tangentes, asíntotas, raíces negativas, curvas ovales, teoría de ecuaciones, método de constantes indeterminadas, clasificación de líneas, y otros muchos quedan casi eclipsados por su admirable creación, la Geometría Analítica. 
Documento I: Discurso leído ante la Real Academia...

¡Gran siglo para la ciencia este, que en un espacio de cincuenta años presenta el Álgebra de Viete y la nueva geometría cartesiana; y gran siglo para la Francia el de Viete, Fermat y Descartes!

Pero el espíritu humano no está aún fatigado de tan colosal aborto, ni ha llegado aún para él el séptimo día, en quede su trabajo sublime descanse.

En Alemania aparecen nuevos átomos de esta serie luminosa de genios, rayo desprendido del que es luz pura y eterna verdad; Kepler, si grande como astrónomo grande también como geómetra; Byrge, que inicia la teoría logarítmica; Tschirnhausen, célebre por sus trabajos sobre la teoría de ecuaciones y por sus estudios sobre las cáusticas; Mercator o Kauffmann, que inicia la teoría de las series; y para abreviar interminables enumeraciones, Leibnitz, fecundísimo y profundo matemático, creador del cálculo del infinito, figura gigantesca de esas que al través de los siglos se alzan en el camino de la historia, señalando a la humanidad el nuevo derrotero que en su penosísima, pero sublime peregrinación, ha de seguir.

Separada del continente con desdeñoso orgullo, como si creyera que su energía sajona y su severo genio deben evitar todo familiar trato con la veleidosa raza latina, y solo en aquella y en este, fundar su gloria, Inglaterra ha sido fecunda en grandes geómetras, y ha igualado y escedido a veces a las naciones continentales; que estudios rudos y severos, cuadran a su rudo y severo carácter. Anderson, discípulo de Viète; Harriot, rival de Viete y de Descartes; Neper, que inventa los logaritmos; Briggs, que los perfecciona; Gregori, que en la óptica y en la geometría es competidor de Newton; Neil, que es el primero que halla una curva rectificable; Oughtred, cuyas obras han sido clásicas en Inglaterra por mucho tiempo; Wren, que rectifica la cicloide; Barrow, precursor de Leibnitz, pues introduce en sus cálculos, representándolos por $e$ y $a$, los incrementos infinitamente pequeños de las abscisas y de las ordenadas, y halla su relación; Milord Brouncker, que descubre las fracciones continuas, y las aplica a la medida del área de la hipérbola y del círculo, y prepara el descubrimiento del cálculo integral, espresando analíticamente los elementos diferenciales de las áreas; el célebre Wallís, geómetra de primer orden, autor de la Aritmética de los infinitos y de la teoría de las series, que mide magnitudes curvilíneas, y aplica el análisis a los indivisibles de Cavalieri; Simpson, que divide con Girard la gloria de haber adivinado los porismos; y por fin Newton, geómetra inmortal, creador del cálculo de las fluxiones, que con Leibnitz penetra en los sublimes misterios del infinito, y por no dividir con nadie su nueva gloria, solo se eleva a los espacios 
celestes, y de Dios recibe, espíritu semi-divino, el secreto de la atracción de los mundos.

¡Qué siglo tan admirable esté del álgebra de Viete, la geometría de Descartes y el cálculo de Newton y Leibnitz!

Gran siglo, sí, para Europa el siglo XVII; más ¿qué ha sido para nuestra España? ¿Qué descubrimiento analítico, qué verdad geométriea, qué nueva teoría lleva nombre español? ¿Quiénes los rivales de Viete, de, Fermat, de Pascal, de Descartes, de Harriot, de Barrow, de Brouncker, de Wallis, de Newton, de Huygens, de Gregorio de San Vicente, de Leibnitz, de los Bernoulli? Yo los busco con ansia en los anales de la ciencia, y no los encuentro; paso impaciente de una a otra historia, por si hallo al fin, en alguna, reparación al desdeñoso e irritante olvido en que todas nos dejan; y en todas ellas, bien que se eche de ver la nacionalidad del escritor por las cariñosas predilecciones que a sus compatriotas muestra, aparecen los nombres de Francia, Italia, Inglaterra, Alemania, Bélgica, Flandes y Holanda, y en todas se paga tributo de respeto y admiración a los grandes geómetras; pero en ninguna encuentro a nuestra España. Y cierro con enojo historias extranjeras, y a historias nacionales acudo, esperando siempre hallar lo que jamás por desdicha encuentro.

Abro la Biblioteca hispana de D. Nicolás Antonio, y en el índice de los dos últimos tomos, que comprenden del año 1.500 al 1.700 próximamente, tras muchas hojas llenas de títulos de libros teológicos y de místicas disertaciones sobre casos de conciencia, hallo al fin una página, una sola, y página menguada, que a tener vida, de vergüenza se enrojeciera, como de vergüenza y de despecho se enrojece la frente del que, murmurando todavía los nombres de Fermat, de Descartes, de Newton, de Leibnitz, busca allí algo grande que admirar, y solo halla libros de cuentas y geometrías de sastres.

Cuánto me duele señores, pronunciar frases tan duras, no hay para qué encarecerlo, que todos los que me oigan lo comprenderán por la penosa impresión que en ellos causen; más la verdad nunca debe ocultarse, y si alguna vez arranca al alma un grito de dolor, ¿qué importa? Es el enrojecido hierro que muerde en la sangrienta llaga, es el provechoso dolor del cauterio purificador de vieja podredumbre.

Si en el siglo XVII no hemos tenido grandes geómetras, causa sin duda habrá para ello, y nos importa investigar cuál pueda ser, para prevenir en lo futuro males que todos debemos deplorar; no es sin embargo mi ánimo escudriñarla hoy, porque estudio sería este que me llevaría demasiado lejos, pues tal vez en ciencias muy distintas de aquellas a que esta Academia consagra sus tareas, habría de buscarse la solución de 
este importante fenómeno histórico.

Basta para mi objeto consignar el hecho, y recomendarlo a la meditación de los hombres pensadores. Y cuenta, señores, que causa, y causa esterna ha existido; porque suponer en nuestra España incapacidad radical y congénita, verdadera impotencia de raza para el estudio de las matemáticas puras, sería grande injusticia y audaz calumnia al genio poderoso y fecundo de este noble suelo. La patria de tantos y tan sublimes poetas, de tantos y tan admirables artistas, de filósofos tan profundos y de tan ilustres pensadores; la patria de Núñez, de Omerique y de D. Jorge Juan, no es digna de tal afrenta; raza en la que la valentía del pensamiento, el vuelo de la imaginación, la fuerza del querer, llegan al estremo a que en nuestra raza han llegado, no es, no debe ser impotente para una ciencia que solo estas humanas facultades y no otras sobrenaturales energías requiere. Y pues no nos ha sido dado alcanzar en la ciencia de Descartes y de Newton glorias que hemos recojido abundantemente en otros ramos del saber, contra algún obstáculo se habrá estrellado sin duda el genio nacional, e importa mucho conocerlo, repito, para evitarlo en lo futuro, si ya desapareció, para acabar de destruirlo si aún quedan restos, para que llegue al fin el día en que se borre la mancha que en el siglo XVII, siglo del despotismo y de la intolerancia, cayó sobre nuestra historia.

Mancha, y mancha vergonzosa repito, porque no basta que un pueblo tenga poetas, pintores, teólogos y guerreros; sin filósofos y sin geómetras, sin hombres que se dirijan a la razón, y la eduquen y la fortifiquen y la eleven, la razón al fin se debilita, la imaginación prepondera y se desborda, hasta el sentimiento religioso se estanca y se corrompe: y si por un vigoroso esfuerzo, pueblo que a tal punto llegue no restablece el armónico equilibrio que entre las facultades del alma humana debe siempre existir, morirá, como mueren los pueblos que se corrompen y se degradan, y hasta aquello mismo que fue en otro tiempo su gloria, será en_ sus postreros instantes su vergüenza y su tormento.

Hé aquí, señores, cuanto de la historia de las matemáticas en España durante el siglo XVII puedo decir; más antes de pasar al siglo XVIII debo, a fuer de imparcial, citar aquí un nombre, pero uno solo, nombre ilustre, más que por sus obras desgraciadamente incompletas, por el verdadero y profundo talento que revelan. Me refiero al geómetra Sanlucarense, Hugo Omerique, que publicó en 1689 la primera parte de una obra de análisis geométrica, y que mereció igloria envidiable! las alabanzas del gran Newton. La segunda parte de este libro no llegó a publicarse, la historia del geómetra andaluz me es absolutamente desconocida, y su nom- 
bre, que brilla un punto, desaparece bien pronto, cosa natural en aquellos calamitosos tiempos de Carlos II.

El siglo XVIII es para las Matemáticas el complemento natural del siglo XVII; los grandes geómetras de este último continuan sus admirables trabajos en toda la primera parte de aquel, y uno y otro siglo se disputan la altísima honra de poseer a los Bernoulli, a Newton y a Leibnitz, como si uno solo no bastase a contener tanto genio y tanta gloria. Enumerar aquí, siquiera de pasada, los grandes descubrimientos matemáticos del siglo XVIII, los grandes geómetras que lo ilustran, las nuevas y elevadas teorías que aparecen, los infinitos horizontes que se abren al pensamiento, los portentos que se realizan, merced al eficacísimo auxilio de estas tres palancas de incalculable poder, el Álgebra, la Geometría Cartesiana y el Cálculo, fuera empresa no solo superior a mis fuerzas, que son bien débiles, sino a las de quien de atleta se precie.

La teoría de ecuaciones, la nueva geometría, el cálculo diferencial y el de las fluxiones, así como sus inversos, las series, el nuevo método de los límites, la teoría de la eliminación, el nuevo análisis combinatorio, las fracciones contínuas y el cálculo de probabilidades, unos se crean, otros se perfeccionan, otros se desarrollan, y todos juntos forman la ciencia moderna, riquísima y hasta exuberante en sus infinitos desarrollos y detalles, armónica en sus múltiples y variadas relaciones internas, profunda y filosófica por los grandes principios generadores que la sintetizan, y que tienden a elevarla a su final y suprema unidad. Y a esta magnífica obra, todas las grandes naciones de Europa, cuál mas cuál menos, todas menos una, ya sabemos cuál, no hay para qué nombrarla, concurren según su propio genio y sus especiales condiciones de carácter.

En vano algunos geómetras, la mayor parte de segundo orden, apegados a los antiguos métodos, se muestran hostiles, por pasión quizá, o tal vez por más ruines móviles, a la reforma; las nuevas teorías triunfan al fin, como triunfará siempre toda idea grande y fecunda, de la resistencia que le opongan viejos intereses y bastardas ambiciones, por más que encuentren aquellos y estas para su ingrata obra, apoyo firmísimo en la ignorancia y en la rutina, verdadera inercia del espíritu, pero que al fin cede al vigoroso impulso del genio o a la ley inflexible de la necesidad.

Y así, mientras algunos combaten los cálculos de Leibnitz y Newton; mientras otros, sin rechazar las modernas invenciones, antes bien comprendiendo sus ventajas sobre la ciencia antigua, continuan sin embargo sus trabajos geométricos, por hábito sin duda, según la manera de Euclides y de los maestros griegos, jóvenes y brillantes analistas siguen animosos a los grandes matemáticos del siglo precedente, y perfeccionan las 
nuevas teorías y los nuevos métodos creados por aquellos.

En Inglaterra, Taylor, Mac-Laurin, Cotes, Simpson, Landen, Stirling, Campbell, Waring, Halley, el ginebrino Fatio, Moivre, francés de nacimiento, pero que huyó de su patria al revocarse el edicto de Nantes, y encontró patria y gloria en la libre Inglaterra, y otros muchos, -aunque inferiores en casi todas las cuestiones de cálculo integral a sus rivales del continente, y no por falta de genio, sino por sobra de orgullo, -eminentes geómetras sin embargo.

En Italia, Grandi, Ricati, Fagnani, Mascheroni, Manfredi, y el inmortal Lagrange, uno de los genios mas grandes que hayan existido en las ciencias matemáticas.

En Francia, La Hire, aunque más bien pertenezca este geómetra al siglo anterior, Vandermonde, Clairaut, Varignon, Fontaine, Rolle, el célebre Monge, el ilustre Bezout, y por último el enciclopedista D’Alambert y el astrónomo Laplace, émulos ambos del piamontés Lagrange.

En Alemania, Goldbach, Cramer, Herman, Walfio, y el inmortal Euler, genio potente, digno heredero de la gloria de Leibnitz.

Todos estos geómetras y otros muchos que pudiera citar, si no crean la ciencia moderna, porque creada la encuentran, la perfeccionan y completan, y a tal punto la elevan a impulsos de su espíritu creador, que momentos hay en que la historia, asombrada ante los prodigios que realizan, se pregunta dudosa si no es superior al siglo de Viete, Fermat, Descartes, Newton y Leibnitz el siglo de los Bernoulli, Lagrange, D'Alambert, Euler y Laplace,

Otro siglo más de gloria para Europa; otro más de silencio y abatimiento para nuestra España.

Cierto es, Señores, que en las ciencias aplicadas, en las que como la mecánica, la astronomía, la geodesia, la navegación, son las matemáticas puras auxiliar poderosísimo, y tanto que hasta se designan aquellas con el nombre de matemáticas aplicadas o mistas, hay dos nombres ilustres y de reputación europea que yo debo recordar hoy, siquiera por dar un rayo de luz a cuadro tan sombrío: son estos Don Antonio Ulloa y el insigne D. Jorge Juan.

Yo reconozco el profundo saber de ambos marinos, y aprecio en lo que valen sus interesantes trabajos geodésicos; yo sé que la célebre obra del último, titulada Exámen marítimo teórico práctico, obra verdaderamente clásica, ha sido única en Europa por muchos años, y ha recibido el honor de ser traducida y comentada en varias lenguas.

Yo pronuncio con orgullo, con legítimo orgullo, el nombre de Don Jorge Juan, y admiro, en fin, esta magnífica figura, honra y prez del ilustre cuerpo de Marina. 
$\mathrm{Al}$ nombre de éstos dos insignes varones debo unir aún en este respetuoso recuerdo otro más: el de D. Gabriel de Ciscar.

Pero estos tres nombres que acabo de citar no disminuyen la fuerza, inquebrantable por desgracia, del severo fallo que sobre el período que reseño lanza la historia.

Porque yo, Señores, para no dar a este trabajo más estensión de la que por su naturaleza debe tener, solo me ocupo hoy, como al principio dije, de las Matemáticas abstractas, ciencia pura, que estudia las leyes soberanas de estas dos categorías de la razón, la cantidad y el orden, y que ha sido la ciencia de Pitágoras, de Platón, de Euclides, de Arquímedes, de Apolonio, de Diofanto, de Ben-Mohamed, de Tartaglia, de Viete, de Fermat, de Descartes, de Harriot, de Caballieri, de Newton, de Leibnitz, de Lagrange, de D'Alambert, de Euler, de Laplace, de Gauss, de los Bernoulli, de Poisson, de Poinsot, de Fourier, de Legendre, de Jacobi, de Cauchy, de Lejeune-Dirichlet, de Abel; icuánto, nombre ilustre! Yo reseño pues la historia de la ciencia, no la de sus aplicaciones; y la ciencia es la verdad abstracta, no la utilidad material que en la geodesia, en la mecánica o en la navegación pueda proporcionar al físico, al mecánico o al navegante aquel conocimiento puro; y así solo consigno los nombres de los geómetras que han estudiado la ciencia por la ciencia, la verdad por la verdad, y porque es luz que la razón ansía, como ansía el ciego la esplendente luz del sol; y el que en este concepto afirme que hemos tenido un geómetra, siquiera uno, en todo el siglo XVIII, famoso descubrimiento hará si prueba lo que afirma.

Amarga, tristísima verdad, bien lo conozco y lo siento, pero gran verdad también, y fuerza es repetirla para que perdamos ilusiones halagüeñas, que solo pueden servir para hacer mayor el daño. Angustiosas reflexiones se agolpan a mi mente al recordar este nuestro. lastimoso atraso, y atraso crónico, en uno de los ramos del saber que más glorias han dado a la época moderna, y que tanto contribuye a vigorizar las más nobles facultades del alma; al ver cómo pasa uno y otro siglo, el XVI, el XVII, el XVIII, y ni un solo geómetra español aparece, no ya en primeralínea, que fuera mucho pedir para tan gran postración, pero ni aun en segunda siquiera; como si viciada esta raza durante siglos enteros necesitáramos siglos también para arrojar el virus que en nuestra sangre inoculara una generación ciega y fanática.

Pero reflexiones son estas que me llevarían muy lejos, y debo ya pensar en poner término a mi largo y penoso trabajo.

Llega al fin nuestro sido XIX, y con verdad y con legítimo orgullo podemos decir, que ni en grandes geómetras, ni en magníficos descu- 
brimientos es inferior al siglo que le precede. Nombres ilustres tiene, es lo difícil hallarlos, sino antes bien no olvidar alguno en la enumeración, por estensa que sea.

Carnot, célebre autor de la geometría de posición. Fourier, que perfecciona el cálculo integral. El ilustre Poisson, cuyos admirables trabajos le colocan a la altura de los primeros analistas. Legendre, que escribe la teoría de los números y la de ecuaciones, y crea en gran parte la de la funciones elípticas. Lejeune-Dirichlet, que muestra en sus descubrimientos sobre los números y las integrales definidas la profundidad de su talento. Poinsot, que con su claro ingenio y su admirable estilo esclarece los puntos más abstractos y oscuros, entre otros, por ejemplo, la rotación alrededor de un punto, las secciones angulares, la teoría de los números, y la de los momentos por la sustitución de los pares a aquella idea abstracta. El insigne Jacobi, que ejercita su creadora inteligencia en el análisis combinatorio de las determinantes, en la teoría de las funciones elípticas, y en el cálculo integral. El gran geómetra noruego Abel, que hubiera sido el Newton del Norte al decir de un escritor, y que muere en la miseria a los 27 años, abandonado de todos y por todos olvidado, dejando sin embargo en lo que de sus' obras se conserva, rasgos que prueban la fuerza creadora de una noble inteligencia. Cauchy, genio profundo, geómetra eminente, que perfecciona la teoría de las series, dándoles un rigor que antes nunca alcanzaron, crea casi la teoría moderna de las imaginarias, perfecciona y desarrolla el cálculo integral, halla el de resíduos, cultiva con gran éxito el análisis combinatorio y simbólico, y en la teoría de los números, y en la de ecuaciones, y aun en la misma Geometría, deja brillantes rasgos de su felicísimo ingenio; de suerte que no hay punto ni materia en la ciencia que no deba algo y aun mucho, y aun todo, al gran matemático francés, que solo con su magnífica teoría de la luz hubiera hecho su nombre inmortal. Lamé, autor de la teoría matemática de la elasticidad, de la del calor, de la de las funciones trascendentes inversas, y que demuestra el célebre teorema de Fermat, por la teoría de los números complejos. Poncelet, que escribe en las prisiones de Rusia su gran obra de las propiedades proyectivas de las figuras. Chasles, que sin contar otros trabajos de gran valor, crea casi la moderna geometría, y resuelve sobre cónicas problemas admirables. Sturm, autor del célebre teorema que lleva su nombre. Liouville, decano de la ciencia, fecundísimo y profundo matemático, cuyos innumerables trabajos en vano intentaria recopilar, pero de los que he de citar tres magníficas $\mathrm{Me}$ morias sobre métodos de integracion, porque en ellas se encuentra parte de los perdidos descubrimientos de Abel. El célebre Gauss, cuyas inves- 
tigaciones aritméticas, Memorias sobre formas cuadráticas y cálculo de probabilidades, son obras clásicas. Hermite, que resuelve las ecuaciones de quinto grado por las funciones elípticas. Y en fin, señores, Galois, Terquen, Catalan, Serret, Bertrand, Briot, Bouquet, Wantzel, Robillier, Binet, Brianchon, Gergonne, Dandelin, Quetelet, Wronski, Sylvester, Salmon, Boole, Cayley, Yvory, Roberts, Moebius, Hesse, Joachim-Sthal, Kummer, Eisenstein, Aronhold, Baltzer, Plucker, Borchardt, Kronecker, Ruffini, Libri, Cremona, Trudi, Novi, Faa-di-Bruno, Padula, Rubini, Janni, Betti, Beltrani, Battaglini, y tantos y tantos otros, que yo no podría citar sin hacer interminable esta Memoria, pues solo consigno aquí aquellos nombres que en tropel y sin orden acuden a mi mente: todos ellos se han mostrado dignos herederos de los grandes geómetras de los siglos XVI, XVII y XVIII, y han enriquecido y elevado la ciencia hasta tal punto, que si por estraño prodigio hoy volvieran a la vida, Descartes, Fermat, Newton y Leibnitz, tendrían mucho que aprender antes que comenzasen a inventar.

Nuestro país, que aspira afanoso a su regeneración en todas las esferas, no podia ser estraño a este gran movimiento científico de Europa, y yo debo consignar que, aparte de los relevantes servicios prestados a la ciencia por esta ilustre Academia, y que por sabidos es inútil repetir, a los cuerpos facultativos, así militares como civiles, y a sus Escuelas especiales se debe ese gran adelanto en los estudios matemáticos que se nota en España de algunos años acá. No puede, en verdad, gloriarse nuestro país de ningún importante descubrimiento, porque cuando tan rezagada queda una nación, harto hace con alcanzar a las que en tres siglos la aventajan; pero el porvenir es suyo, su voluntad será enérgica, el campo del saber es infinito, y génios tendrá cuando libre de fatales trabas, y conquistada la libertad filosófica, que es la libertad del pensamiento, se lance de lleno al estudio de esta gran ciencia que dió a Descartes, a Newton y a Leibnitz nombre inmortal. Y esta es, señoreŝ, la historia de las matemáticas en nuestra pátria.

Pero no: si, prescindiendo de aquellos siglos en que la civilización arábiga hizo de España el primer país del mundo en cuanto a la ciencia se refiere, solo nos fijamos en la época moderna, y comenzamos a contar desde el siglo XV, bien comprendeís que no es esta, ni puede ser esta en verdad, la historia de la ciencia en España, porque mal puede tener historia científica, pueblo que no ha tenido ciencia. La imperfecta relación que habeis oido, es resúmen histórico de la ciencia matemática, sí; pero en Italia, en Francia, en Inglaterra, en Holanda, en Alemania, en Suiza, que es donde renace la geometría, donde se crea el álgebra, la geometría carte- 
Documento I: Discurso leído ante la Real Academia...

siana, la teoría de ecuaciones, el análisis algebraico, la teoría de los números, los cálculos del infinito, el análisis indeterminado, el cálculo combinatorio, la moderna geometría trascendente y la teoría de las curvas: es la historia de la ciencia allá donde hubo un Viete, un Descartes, un Fermat, un Harriot, un Wallis, un Newton, un Leibnitz, un Lagrange, un Cauchy, un Jacobi, un Abel; no es la historia de la ciencia, aquí donde no hubo mas que látigo, hierro, sangre, rezos, braseros y humo.

$\mathrm{Y}$ yo he tenido que referir la historia de las matemáticas, allá, para probar que no la hay aqui, $y$ para probarlo, señores, con la elocuente voz de los hechos, demostración ruda pero firmísima, contra la cual se estrellan impotentes, sofismas, alharacas y declamaciones, he necesitado buscar la filiación de cada verdad, el origen de cada teoría, el nacimiento de cada idea, el autor de cada descubrimiento, y después los hombres que desarrollan y perfeccionan aquellos descubrimientos y teorías, formando de esta suerte la ciencia moderna en toda su magnífica riqueza; y he necesitado todo esto para poder decir sin remordimiento y sin temor: la ciencia matemática nada nos debe: no es nuestra; no hay en ella nombre alguno que labios castellanos puedan pronunciar sin esfuerzo.

La resolución de las ecuaciones de tercero y cuarto grado se debe a Ferri, a Tartaglia, a Cardan y a Ferrari, italianos.

El álgebra a Viete, francés.

La teoría de ecuaciones, al mismo Viete, francés,y a Harriot, inglés.

La geometría analítica, a Descartes, francés también.

El cálculo diferencial a Newton y Leibnitz, inglés el primero, aleman el segundo.

La teoría de los números y el análisis indeterminado, a Fermat y Bachet de Meziriac, franceses ambos.

Las fracciones contínuas a Brouncker, inglés.

Los logaritmos a Neper, inglés tambien,

La geometría superior a Desargues, francés.

Las series a Wallis y Mercator, inglés el primero, alemán el segundo.

El cálculo integral a Leibnitz, Newton y los Bernoulli. Al francés Monge, la geometría descriptiva.

El cálculo de variaciones, al piamontés Lagrange.

Y después amplían, desarrollan y perfeccionan estas teorías, o crean otras nuevas, numerosos e insignes geómetras, todos extranjeros a nuestra patria.

De esta suerte en la edad moderna, la Italia desgarrada por españoles, franceses y alemanes; la Francia dividida y ensangrentada por sus guerras civiles y religiosas; Alemania entregada a todos los horrores del 
encarnizamiento socialista y religioso, y al azote de guerras nacionales; la Holanda, la Bélgica, Flandes y los Paisés-Bajos gimiendo bajo: el peso de nuestra feroz dominación; Inglaterra, que ve subir a su rey a un cadalso, y sufre, como el resto de Europa, las convulsiones de las grandes luchas religiosas.

Todos estos pueblos, entre guerras y sangre, y terribles sacudimientos, conservan entera y vigorosa su razón, y de entre el caos y las ruinas se alzan genios potentes, nobles inteligencias, profundos filósofos y grandes geómetras; y en nuestra España, invencible y poderosa, dueña del nuevo mundo, y aspirando a dominar el antiguo, tranquila, relativamente al resto de Europa, en el interior, temida fuera, con su unidad política y su unidad religiosa, solo se conservan puros, y no siempre, la imaginación y el sentimiento; pero la razón, la facultad más noble del ser que piensa, languidece y decae, y con ella todo languidece y muere al fin.

Contraste singular, sobre el que omito reflexiones, pero sobre el cual no puedo menos de llamar vuestra atención. Los grandes hechos históricos encierran provechosa enseñanza, y lecciones hemos recibido en lo pasado, que, si hoy somos capaces de comprender, pueden servirnos grandemente para el porvenir.

$\mathrm{Y}$ ahora, señores, descrita ya a grandes rasgos la historia de las Matemáticas puras, y puesta de relieve por la comparación con otros países, nuestra deplorable decadencia desde el renacimiento, que fue para España más bien morir que renacer, hasta principios de este siglo, debiera dar por terminada mi tarea; pero hay un punto de suma importancia, íntimamente enlazado con el tema de mi discurso, y sobre el cual he de presentar, siquiera de pasada, algunas reflexiones que recomiendo a la meditación de vuestro elevado pensamiento.

Hay, señores, quien imagina, y personas ilustradas y respetables son por singular inconsecuencia de esta opinión, que la gran importancia, la verdadera utilidad, el incuestionable valor de las Matemáticas puras, solo reside en la aplicación que de los principios abstractos de la ciencia pueda hacerse a la física, a la geodesia, a la mecánica, y principalmente a la industria; y que toda verdad científica, por elevada que sea, a la que no corresponda una utilidad práctica, y por decirlo así tangible, es vana gimnasia de la razón, fugaz relampaguear de la fantasía, juego pueril que para nada sirve, descubrimiento estéril que, sin daño del bien común, pudo quedar algunos siglos más envuelto entre las sombras, de las que le arrancó por caprichoso entretenimiento algún desocupado geómetra. 


\section{Documento I: Discurso leído ante la Real Academia...}

Los que así juzguen y en tan poco tengan a la ciencia, no encontrarán grave mal en nuestro atraso durante los siglos XVI, XVII, XVIII y XIX, ni en esa falta de grandes geómetras que tanto en esta larga Memoria he deplorado, y se darán por satisfechos con que de la ciencia abstracta solo sepamos hoy, y solo hayamos sabido, lo puramente preciso para las aplicaciones materiales.

¡A qué, dirán, acumular volúmenes sobre volúmenes, henchidos de caprichosas notaciones, semicabalísticas fórmulas, enmarañadas teorías e ininteligible fraseología!

¿Sirve todo ello para dar a la industria un nuevo engranaje que economice rozamientos, un sistema de puentes preferible a los conocidos, una disposición ventajosa para el hogar de la locomotora, u otra pila mas para el telégrafo eléctrico? ¿En una palabra, esa verdad abstracta se traduce en algo práctico, satisface necesidades materiales de nuestra moderna sociedad? Si así es, reconocemos su importancia, dirán los que profesan la opinión que voy a combatir, si lo contrario fuere, la negamos sin titubear.

La cuestión, presentada de este modo, puede ya formularse con mas claridad en estos nuevos términos:

¿La importancia de la ciencia reside solo en sus aplicaciones, o a más de estribar en ellas, depende del valor que la ciencia tenga por sí misma, dado que tenga alguno?

Porque yo, señores, desdeño cierta tímida defensa que de las verdades abstractas pudiera hacerse, defensa fundada en que lo que es hoy puramente ideal, puede mañana adquirir gran importancia práctica, no, yo presento la dificultad de frente, y sin eludirla, ni temerla, de frente también la acometo, y seguro estoy que ha de quedar vencida: yo supongo, por lo tanto, que la ciencia pura jamás tenga la aplicación material que se supone, y aun partiendo de esta desventajosísima hipótesis, he de sostener el estudio de la ciencia por la ciencia , sin fin alguno utilitario, o más bien, dando a la palabra utilidad, no el sentido materialista y grosero de otros tiempos y de ciertas escuelas, sino el más científico y único aceptable de la ciencia moderna, yo he de probar, repito, que toda verdad abstracta es grandemente útil y a todas luces provechosa.

La opinión que combato, es fórmula menguada y repugnante del más, embrutecedor materialismo; tal doctrina desconoce por completo las más nobles, las más puras, las más elevadas facultades del hombre; le mutila torpemente, y sin piedad, le reduce a inmunda bestia. No, señores, el hombre, ser complejo, se halla dotado de muchas y diversas facultades; energías latentes que tienden a desarrollarse, y en cuyo desarrollo si- 
multáneo y armónico consiste la perfección humana. Y de estas energías que buscan satisfacción, es decir, modo y manera, y condiciones y elementos propios para su desarrollo, nacen las varias necesidades del individuo y de la sociedad.

Tiene necesidades materiales, es cierto, y para satisfacerlas trabaja la industria material, y rechinan las fábricas, silba la locomotora, hierve el vapor; pero tiene necesidades de un orden más elevado; ama lo bello, y ama la verdad, y por eso apenas sale del estado salvaje, crea el arte y busca la ciencia. Así, señores, es la ciencia eminentemente útil, no de una manera indirecta por sus aplicaciones, sino directa e inmediata, porque directa e inmediatamente y por su propia virtud, satisface altísimas necesidades humanas, y del mismo modo que el cuerpo busca el pan de cada día, busca el alma, hambrienta de belleza y de verdad, algo que satisfaga las aspiraciones a lo infinito de su inmortal esencia; busca la verdad repito, por esa misteriosa atracción que entre la verdad y el pensamiento existe, y que hace que la razón vaya tras ella anhelante, y sin ella muera, y con ella viva; y que al hallarla en su esencia divina, se sumerja y se bañe gozosa como en océano de luz.

Esto no escluye la aplicación práctica de la verdad abstracta; más bien puede aquí decirse, "amad a la ciencia por la ciencia, a la verdad por la verdad, que el resto se os dará en añadidura.»

Las verdades ideales de las matemáticas son tan reales, más reales, si me es permitida esta frase, que las del mundo físico, porque es el hombre realidad, más intensa, y más rica, y más elevada, que el mundo de la materia y de los sentidos; y si nadie pone en duda la utilidad de las ciencias naturales, la Física, la Química, por ejemplo, no sólo por sus aplicaciones, sino porque en ellas se estudian las leyes generales de la materia, mal puede negarse la utilidad de las verdades abstractas de las Matemáticas, que son las leyes de dos grandes y universales categorías, la cantidad y el orden; categorías que, como de esencia divina, todo, por decirlo así, lo penetran, y desde la razón humana hasta la última molécula material, desde el infinitamente pequeño al infinitamente grande, en todas partes se hallan, y por do quiera, en el mundo del espíritu o en el mundo físico, sentimos con sublime estremecimiento su divina palpitación.

Y si tal es la importancia y el valor de la ciencia pura, y tan alto puesto ocupa entre los conocimientos abstractos el de estas dos grandes categorías, la cantidad y el orden, ved si con razón deploraba nuestro vergonzoso atraso en la época moderna, si con razón anatematizaba las funestas causas de tanto mal, y si con ánsia debemos todos desear que llegue pronto el día de nuestra completa regeneración científica. Porque 
Documento I: Discurso leído ante la Real Academia...

no lo dudeis, entre las grandes facultades del espíritu, hay una necesaria armonía, y pueblo en que esta armonía se turba, camina indefectiblemente a su ruina. Si pierde el sentimiento religioso, o a bárbaras o atrasadas religiones se entrega, le espera la inmoralidad, la descomposición y la muerte, en un porvenir más o menos lejano; si es insensible al sentimiento artístico y poético, el materialismo le devorará al fin; pero del mismo modo, si no ama la ciencia pura, y con ella fortifica su razón, caerá fatalmente en vergonzoso embrutecimiento, y desdeñado por todos, estraño a la vida del pensamiento, sufrirá la pena del olvido, triste muerte de todo pueblo que no ha sabido conquistar su inmortalidad en la historia.

No temais tanto mal ni tan vergonzoso fin para nuestra España; ella ha sabido siempre, en los supremos momentos, alzarse desde la mayor postración a las mayores glorias, y ella sabrá ganar el tiempo perdido, conquistando bien pronto honroso puesto entre las naciones de Europa, mientras llega el día, y llegará, en que la patria de Murillo, de Cervantes, de Luis de Vives, de Omerique y de D. Jorge Juan, dé al mundo rivales de Newton y Descartes, y nuevos lauros á nuestra gloriosa historia.

José Echegaray 